\title{
Vorfälligkeitsentschädigung: Pragmatischer Interessenausgleich ist möglich
}

\author{
Streitfragen bezüglich der Vorfälligkeitsentschädigung beschäftigen seit vielen Jahren \\ deutsche Gerichte, weil die Bundesregierung die Berechnung bislang nicht rechtsverbindlich \\ geregelt hat. Eine von zwei Bundesministerien eingesetzte Arbeitsgruppe legte Ende 2018 \\ ihren Abschlussbericht vor. Ein Konsens zur Schadensberechnung und Verbesserung der \\ Transparenz konnte jedoch nicht erzielt werden. Ob und wie der Gesetzgeber nun reagieren \\ wird, ist unklar. Daher werden hier Beispielrechnungen dargestellt, die wichtigsten Streitpunkte \\ untersucht und einige pragmatische Vorschläge diskutiert.
}

\begin{abstract}
Eine Vorfälligkeitsentschädigung dient dem Ausgleich eines finanziellen Schadens, der einem Kreditgeber dadurch entsteht, dass ein Kreditnehmer einen Kredit entgegen den vertraglichen Regelungen vorzeitig tilgt oder mit seinem Schuldendienst pflichtwidrig in Verzug gerät. Ein Schaden, der dem Kreditgeber dadurch entsteht, dass der Kreditnehmer pflichtwidrig die Abnahme eines vertraglich vereinbarten Darlehens verweigert, ist durch eine sogenannte Nichtabnahmeentschädigung zu erstatten. Deren Höhe wird nach denselben durch den Bundesgerichtshof $(B G H)$ im Laufe der Jahre entwickelten Grundsätzen berechnet. ${ }^{1} \mathrm{Ob}$ die Vorfälligkeitsentschädigung gerechtfertigt und angemessen ist, wird seit Jahrzehnten juristisch debattiert, zumal es bis heute keine eindeutige rechtsverbindliche Regelung zur Berechnung der Entschädigung gibt. Verbraucherschützer kritisierten in einer Studie von 2014, dass in knapp zwei Dritteln der untersuchten Fälle die von Kreditgebern geforderte Entschädigung um mehr als 2,5\% über dem Wert gewesen
\end{abstract}

(C) Der/die Autor(en) 2020. Open Access: Dieser Artikel wird unter der Creative Commons Namensnennung 4.0 International Lizenz (http:// creativecommons.org/licenses/by/4.0/deed.de) veröffentlicht.

Open Access wird durch die ZBW - Leibniz-Informationszentrum Wirtschaft gefördert.

1 Aufgrund der weitgehenden Übereinstimmung der Problematik von Vorfälligkeitsentschädigung und Nichtabnahmeentschädigung wird im Folgenden nur noch die Vorfälligkeitsentschädigung genannt.

Stefan Best, Dipl.-Volkswirt, ist Dozent am Fachbereich Wiesbaden Business School der Hochschule RheinMain in Wiesbaden. sei, der sich bei Anwendung der durch die Rechtsprechung vorgegebenen Verfahren ergeben hätte. ${ }^{2}$

Ein weiterer Grund für die Auseinandersetzungen ist der seit Jahren andauernde Rückgang des Zinsniveaus. So sind nach Angaben der Bundesbank allein zwischen April 2011 und September 2019 die Zinssätze im Neugeschäft für besicherte Wohnungsbaukredite an private Haushalte je nach Dauer der Zinsbindung um 2,6 bis 3,3 Prozentpunkte gefallen. Auch wenn sich der Abwärtstrend verlangsamt hat, kann die Vorfälligkeitsentschädigung auch bei geringeren Zinsveränderungen je nach Höhe der Restschuld, Restlaufzeit des Darlehens, Tilgungsform etc. gerade bei gewerblichen und Immobilienkrediten mehrere 1000 Euro betragen. Zudem ist das Thema auch bei steigenden Zinsen bedeutend, da die Behandlung eines Vorfälligkeitsnutzens für den Kreditgeber ebenfalls umstritten ist.

Arbeitsgruppe Vorfälligkeitsentschädigung bleibt praktisch ergebnislos

In der Vergangenheit hatte der Gesetzgeber es stets abgelehnt, Details zur Vorfälligkeitsentschädigung rechtsverbindlich zu regeln. ${ }^{3}$ Der Bundesrat hatte im Zusammenhang mit dem Finanzaufsichtsrechtergänzungsgesetz

2 Verbraucherzentrale Bundesverband: Vorfälligkeitsentschädigungen: Überprüfung und Bewertung der Angemessenheit und Rechtmäßigkeit von Entschädigungszahlungen von Verbrauchern bei vorzeitig gekündigten Immobilienkrediten, Stand Juli 2014, https://www.vzbv. de/sites/default/files/downloads/Vorfaelligkeitsentschaedigungen_ Untersuchung_vzbv_2014.pdf (13.12.2019).

3 Vgl. Entwurf eines Gesetzes zur Modernisierung des Schuldrechts, 2001, Bundestags-Drucksache 14/6040, S. 255; vgl. Unterrichtung durch die Bundesregierung, Entwurf eines Gesetzes zur Umsetzung der Wohnimmobilienkreditrichtlinie, Bundestags-Drucksache 18/6286; Stellungnahme des Bundesrates und Gegenäußerung der Bundesregierung, 2015, Bundesrats-Drucksache 18/5922, S. 24; vgl. Entwurf eines Gesetzes zur Umsetzung der Wohnimmobilienkreditrichtlinie, 2015, Bundesrats-Drucksache 359/15, S. 9. 
erneut gefordert, „Einzelheiten und Methoden für die Berechnung der Vorfälligkeitsentschädigung bei ImmobiliarVerbraucherdarlehensverträgen" festzulegen. Die Bundesregierung verwies aber auf die von den Bundesministerien für Finanzen sowie für Justiz und Verbraucherschutz im Juli 2016 kurz nach Verabschiedung der Wohnimmobilienkreditrichtlinie eingesetzte gemeinsame Arbeitsgruppe zum Thema „Vorfälligkeitsentschädigung“, deren Ergebnisse zunächst abgewartet werden sollten. ${ }^{4}$ Sie sollte prüfen, ob es weiteren Kodifizierungsbedarf gibt, wie die Vorfälligkeitsentschädigung berechnet werden sollte, und wie mehr Transparenz geschaffen werden kann. ${ }^{5}$ Am 18.9.2018 legte sie ihren Abschlussbericht vor. ${ }^{6}$ Sie konnte jedoch in keiner der maßgeblichen Fragen einen Konsens erzielen, da sich die Positionen der Verbraucher- und der Bankenlobby unversöhnlich gegenüberstanden. Auch bei den beiden als „einvernehmliche Empfehlung“ dargestellten Punkten einer Stichtagsregelung zur Berücksichtigung von Sondertilgungsrechten sowie einer Karenzzeitregelung bezüglich des Abrechnungszeitpunkts, gab es letztlich im Detail keine Einigung.

Nach dem praktisch ergebnislosen Versuch durch die Beauftragung der Arbeitsgruppe, die Berechnung der Vorfälligkeitsentschädigung praktikabler und transparenter zu gestalten, ist unklar, wie es nun weitergehen soll. Dabei sind Verbesserungen durchaus möglich, wenn man akzeptiert, dass ihre exakte Berechnung praktisch unmöglich ist und daher eine faire und praktikable Lösung Kompromissbereitschaft abverlangt.

\section{Die Aktiv-Passiv-Methode}

Die Höhe einer Vorfälligkeitsentschädigung ergibt sich aus dem Unterschiedsbetrag zwischen dem Barwert der noch ausstehenden Zins- und Tilgungszahlungen aus dem Kreditvertrag und der Restschuld zum Zeitpunkt der Beendigung des Kreditvertrags. Ist der Unterschiedsbetrag negativ, spricht man von einem Vorfälligkeitsnutzen, da in diesem Fall der Kreditgeber von einer vorzeitigen Beendi-

4 Vgl. Entwurf eines Gesetzes zur Ergänzung des Finanzdienstleistungsaufsichtsrechts im Bereich der Maßnahmen bei Gefahren für die Stabilität des Finanzsystems und zur Änderung der Umsetzung der Wohnimmobilienkreditrichtlinie - Finanzaufsichtsrechtergänzungsgesetz, 2017, Bundesrats-Drucksache 815/1/16, S. 26-29; vgl. Beschlussempfehlung des Finanzausschusses zu dem Gesetzentwurf der Bundesregierung, Entwurf eines Gesetzes zur Ergänzung des Finanzdienstleistungsaufsichtsrechts im Bereich der Maßnahmen bei Gefahren für die Stabilität des Finanzsystems und zur Änderung der Umsetzung der Wohnimmobilienkreditrichtlinie - Finanzaufsichtsrechtergänzungsgesetz, 2017, Bundestags-Drucksache 18/11774, S. 31.

5 Vgl. Bundesministerium der Finanzen: „Gemeinsame Arbeitsgruppe von BMJV und BMF zum Thema Vorfälligkeitsentschädigung“ vom 21.7.2016.

6 Vgl. Abschlussbericht der Arbeitsgruppe Vorfälligkeitsentschädigung vom 18.9.2018, https://www.bundesfinanzministerium.de/ Content/DE/Standardartikel/Themen/Internationales_Finanzmarkt/ Finanzmarktpolitik/2018-11-06-Vorfaelligkeit.html (13.12.2019). gung des Vertrags profitiert. Die Zahlungen zur Ermittlung des Barwerts werden laufzeitkongruent durch Multiplikation mit sogenannten Zerobondabzinsungsfaktoren (ZBAF) abgezinst. ${ }^{7}$ Als relevante Zinskurve sieht das BGH die von der Bundesbank täglich zur Verfügung gestellten Werte für Pfandbriefe an, was man als einen plausiblen Kompromiss ansehen kann, da es sich um eine vergleichsweise risikoarme und faktisch durchführbare Wiederanlagealternative handelt. ${ }^{8}$ Hierzu veröffentlicht die Bundesbank ebenfalls täglich Zerobondrenditen für Laufzeiten von einem Jahr bis 20 Jahren. ${ }^{9}$ Da diese Zerobondrenditen nur volle Jahre abbilden, müssen für gebrochene Laufzeiten Renditen bzw. ZBAF interpoliert werden. Im Sinne der Einfachheit und Transparenz wäre es sinnvoll, die lineare oder exponenzielle Interpolation vorzuschreiben. ${ }^{10}$ Der Zusammenhang zwischen der als Spot Rate bezeichneten Zerobondrendite und dem entsprechenden ZBAF für eine Laufzeit $t$ lautet: ZBAF $_{t}=1 /\left(1+\text { Spot Rate }_{t}\right)^{t}$.

Anhand eines Beispiels wird in Tabelle 1 die Berechnung einer Vorfälligkeitsentschädigung gezeigt. Entsprechend der dargestellten Vorgehensweise ist der Barwert sämtlicher, vertraglich vereinbarter Zins- und Tilgungszahlungen zu berechnen. Die positive Differenz aus der Summe dieser Barwerte abzüglich der Restschuld ergibt die vom Kreditnehmer zu leistende Vorfälligkeitsentschädigung brutto. Sollte sich hingegen, in einer anderen Zinskonstellation, also typischerweise dann, wenn das Zinsniveau gegenüber dem Zeitpunkt der Festzinsvereinbarung gestiegen ist, eine negative Differenz, also ein Vorfälligkeitsnutzen für den Kreditgeber ergeben, sollte dieser Nutzen entsprechend dem Kreditnehmer zugutekommen. Nach der aktuellen Rechtslage hat jedoch der Kreditnehmer darauf keinen Anspruch. Stattdessen wird der Nutzen lediglich bis zur Gesamthöhe des Schadens angerechnet. Ein den Schaden übersteigender Nutzen verbleibt hingegen beim Kreditgeber. ${ }^{11}$

Das Beispiel soll nun, wie in der Praxis üblich, um die Behandlung des Kreditrisikos und der Verwaltungskosten

7 Die Betrachtung von Geld-Brief-Spannen sollte man aufgrund ihrer in normalen Zeiten geringen Bedeutung für das Ergebnis und im Sinne einer pragmatischen Lösung vernachlässigen. Zu dem Thema vgl. K. Wimmer, P. Rösler: Vorfälligkeitsentschädigung bei vorzeitiger Beendigung von Darlehensverträgen, in: Zeitschrift für Wirtschafts- und Bankrecht, 59. Jg. (2005), H. 40, S. 1876.

8 Vgl. BGH Urteil XI ZR 285/03 vom 30.11.2004.

9 Vgl. Deutsche Bundesbank, https://www.bundesbank.de/dynamic/ action/de/statistiken/zeitreihen-datenbanken/zeitreihen-datenbank/ 759778/759778? listld=www_skms_it04a (13.12.2019).

$10 \mathrm{Vgl}$. H. Hewicker, H. Cremers: Modellierung von Zinsstrukturkurven, Frankfurt School - Working Paper Series, Nr. 165, Frankfurt School of Finance \& Management, Frankfurt a. M. 2011, S. 7 f.

11 Vgl. H.-M. Krepold: Kündigungsrecht, in: H. Schimansky, H.-J. Bunte, H.-J. Lwowski (Hrsg.): Bankrechtshandbuch, 5. Aufl., 2017, § 79, Rn 102; vgl. E. v. Heymann, P. Rösler: Berechnung von Vorfälligkeitsund Nichtabnahmeentschädigung, in: Zeitschrift für Wirtschaftsrecht, 2001, H. 11 , S. 449 
erweitert werden. Die ausstehende Zahlung von 100000 Euro in $t_{2}$ ist mit einem Kreditrisiko behaftet ist. Wenn der Kreditnehmer den Kredit vorzeitig tilgt, entfällt auch die Notwendigkeit, dem Kreditgeber für die Restlaufzeit eine Kompensation für die Risikokosten zu zahlen. Dies gilt ebenso für Verwaltungskosten, die durch die vorzeitige Tilgung entfallen. Risikoprämien sind abhängig von der Bonität des Kreditnehmers und der Besicherung. Für eine transparente und pragmatische Regelung würde es sich anbieten, für typische Kreditarten gewisse Standardisierungen vorzunehmen. So könnten durchschnittliche Risikokosten je nach Kreditart branchenweit aus den von den Banken für die Eigenmittelunterlegung ermittelten Parametern Ausfallwahrscheinlichkeit (PD) und Verlustquote bei Ausfall (LGD) geschätzt und ebenfalls von der Bundesbank oder Bundesanstalt für Finanzdienstleistungsaufsicht (BaFin) veröffentlicht werden. Aus dem Säule3-Bericht der Deutschen Bank lässt sich beispielsweise für durch Immobilien besicherte Forderungen, ohne kleine und mittlere Unternehmen, eine gewogene durchschnittliche Risikoprämie als Produkt aus PD und LGD in dem für Neuvergabe vermutlich noch relevanten Bereich bis zu einem Rating von B- in Höhe von 0,12 \% p. a. ermitteln, die etwas über den oft zitierten von der Rechtsprechung akzeptierten Werten bis $0,06 \%$ liegt. $^{12}$ Ebenso könnte man entsprechende Branchendurchschnitte berechnen.

Die dem Kreditnehmer ersparten Verwaltungskosten sind nach Meinung des BGH unabhängig von der Höhe der Restschuld zu kalkulieren. Bei vertragsgemäßem Verlauf dürften diese von eher untergeordneter Bedeutung sein. So wurden von Gerichten eine geschätzte Ersparnis von 30 Euro bis 60 Euro p.a. als angemessen angesehen. ${ }^{13}$ Der Barwert dieser ersparten Kosten reduziert ebenfalls die zu zahlende Vorfälligkeitsentschädigung. Schließlich steht dem Kreditgeber ein Bearbeitungsentgelt für die Berechnung der Vorfälligkeitsentschädigung zu, wofür je nach Sachlage von Gerichten 250 bis 400 Euro als Schätzwert (im Beispiel 300 Euro) angesetzt werden können. ${ }^{14}$ Gemessen an den Lohnkosten im Bereich Finanzdienstleistungen ${ }^{15}$ dürften infolge der Automatisierung die

12 Vgl. Deutsche Bank: Säule 3 Bericht 2018, S. 96, https://www. db.com/ir/de/regulatorische-veroeffentlichungen.htm (13.12.2019); vgl. P. Rösler, K. Wimmer, V. Lang: Vorzeitige Beendigung von Darlehensverträgen, München 2003, S. 139.

13 Wobei in der Praxis zugunsten der Kreditnehmer auch höhere Beträge bis 120 Euro p. a. angesetzt wurden, vgl. G. Nobbe: Buch 2: Recht der Schuldverhältnisse, Abschnitt 8: Einzelne Schuldverhältnisse, in: H. Prütting, G. Wegen, G. Weinreich (Hrsg.): BGB Kommentar, 13. Aufl., Köln 2018, S. 874; vgl. Abschlussbericht der Arbeitsgruppe Vorfälligkeitsentschädigung vom 18.9.2018, a.a. O., S. 31.

14 G. Nobbe, a. a. O., S. 874.

15 Statistisches Bundesamt: Leitfaden zur Ermittlung und Darstellung des Erfüllungsaufwands in Regelungsvorhaben der Bundesregierung, Dezember 2018, Anhang VI Lohnkostentabelle Wirtschaft, Wirtschaftsabschnitt K, S. 55.
Tabelle 1

Berechnung einer Vorfälligkeitsentschädigung in Euro

\begin{tabular}{lccc} 
& $\mathrm{t}_{0}$ & $\mathrm{t}_{1}$ & $\mathrm{t}_{2}$ \\
\hline Restschuld des Kredits & 96116,88 & & \\
\hline Ausstehende Zahlungen & & 100000,00 \\
\hline $\begin{array}{l}\text { Barwert der ausstehenden } \\
\text { Zahlungen vor Zinsveränderung }\end{array}$ & 96116,88 & \\
\hline $\begin{array}{l}\text { Barwert der ausstehenden } \\
\text { Zahlungen nach Zinsveränderung }\end{array}$ & 98029,60 & \\
\hline $\begin{array}{l}\text { Vorfälligkeitsentschädigung, } \\
\text { brutto }\end{array}$ & 1912,73 & \\
\hline Risikokosten 0,1\% p.a. & & $-96,12$ & $-98,04$ \\
\hline $\begin{array}{l}\text { Barwert der ersparten } \\
\text { Risikokosten }\end{array}$ & $-191,27$ & \\
\hline Ersparte Verwaltungskosten p.a. & & $-60,00$ & $-60,00$ \\
\hline Barwert der ersparten & & \\
Verwaltungskosten & $-118,22$ & \\
\hline Bearbeitungskosten & 300,00 & \\
\hline $\begin{array}{l}\text { Vorfälligkeitsentschädigung, } \\
\text { netto }\end{array}$ & 1903,23 & \\
\hline
\end{tabular}

Das Beispiel: Die Restschuld eines zinslosen und endfälligen Kredits betrage heute $\left(t_{0}\right)$ 96116,88 Euro bei einer vertraglich noch ausstehenden Zahlung von 100000 Euro in zwei Jahren $\left(t_{2}\right)$. Dies entspricht einer Verzinsung von $2 \%$, denn es gilt: $96116,88 * 1,02^{2}=100000$. Die aktuelle Spot Rate sei $1 \%$. Wenn nun der Kreditnehmer vorzeitig lediglich die Restschuld des Kredits ohne Zahlung einer Vorfälligkeitsentschädigung tilgt, entsteht dem Kreditgeber ein Schaden, da er den erhaltenen Betrag nur zu $1 \%$ anlegen kann. Somit entgehen inm $100000-96116,88$ * $1,01^{2}=1951,17$ Euro in $t_{2}$, was einem Barwertrückgang von 1951,17 Euro * $1 / 1,01^{2}=1912,73$ Euro in $t_{0}$ entspricht. Demnach muss der Kreditnehmer neben der Restschuld von 96116,88 Euro auch brutto eine Vorfälligkeitsentschädigung in Höhe von 1912,73 Euro und somit insgesamt 98029,60 Euro zahlen. Durch die Anlage dieses Betrags am Kapitalmarkt zu $1 \%$ für zwei Jahre wird der ursprünglich vereinbarte Zahlungsstrom von 100000 Euro nachgebildet. (Vgl. K. Wimmer, E. Caprano: Finanzmathematik, 7. Aufl., München 2013, S. 182 ff.)

Unterstellt man etwa im Falle einer privaten Immobilienfinanzierung Risikokosten in Höhe von $0,1 \%$ p.a., betragen diese im Beispiel für das 1. Jahr $96116,88 * 0,1 \%=96,12$ Euro und für das 2. Jahr $96116,88 * 1,02 * 0,1 \%=$ 98,04 Euro. Daraus resultiert bei Abzinsung mit $1 \%$ ein Barwert in Höhe von 191,27 Euro, der die zu zahlende Vorfälligkeitsentschädigung reduziert.

Quelle: eigene Berechnungen.

ersparten Verwaltungskosten p.a. eines störungsfrei verlaufenden Kredits unter 120 Euro und das Bearbeitungsentgelt pro Berechnung unter 250 Euro liegen.

Angesichts der für die Berechnung der Vorfälligkeitsentschädigung benötigten Komponenten, also die für die Abzinsung zu verwendende und eine entsprechende Wiederanlage implizierende Zinskurve, Annahmen über die Ausübung von Sondertilgungsrechten, sowie die Höhe der Risiko-, Verwaltungs- und Bearbeitungskosten, lässt 
sich der durch vorzeitige Tilgung bzw. Nichtabnahme entstehende Schaden nicht exakt kalkulieren, sondern lediglich auf plausible Weise und nur mit technischer Hilfe schätzen. Daher ist die Verwendung von Schätzungen zur Schadensberechnung unumgänglich, aber auch juristisch gemäß § 287 Zivilprozessordnung anerkannt. ${ }^{16}$

\section{Vergleich mit der Aktiv-Aktiv-Methode}

In seinem Urteil vom 1.7.1997 vertrat der BGH die Auffassung, dass eine Bank den ihr durch die vorfällige Rückzahlung oder pflichtwidrige Nichtabnahme eines Darlehens entstehenden Schaden auf unterschiedliche Weise berechnen kann. ${ }^{17}$ Bei der Aktiv-Passiv-Methode wird die Entschädigungszahlung in einer Summe durch den Schaden ermittelt, der dadurch entsteht, dass das vorzeitig erhaltene Kapital nicht zum ursprünglich vereinbarten Vertragszins, sondern zum aktuellen Kapitalmarktzins angelegt werden kann. Bei der Aktiv-Aktiv-Methode ergibt sich die Vorfälligkeitsentschädigung aus der Summe aus Zinsmargenschaden (entgangener Nettogewinn vor Steuern) und Zinsverschlechterungsschaden (Schaden aus der Wiederanlage des vorzeitig erhaltenen Kapitals zu einem aktuellen Kapitalmarktzins unterhalb der ursprünglichen Refinanzierungskosten des Kreditgebers). Ob die Bank das Darlehen tatsächlich fristenkongruent refinanziert hat, oder stattdessen ein Zinsrisiko aus einer Fristentransformation eingegangen ist, ist für die Betrachtung unerheblich. Entscheidend für die Berechnung des Zinsmargenschadens ist die Höhe der aus dem Kundengeschäft resultierenden Marge (Konditionsbeitrag), da die Bank Zinsrisiken auch jederzeit unabhängig vom Kundengeschäft eingehen und so einen Strukturbeitrag erzielen kann. ${ }^{18}$

Zwar führen beide Verfahren bei korrekter Anwendung zu demselben Ergebnis. Allerdings ist die Aktiv-Passiv-Methode in der Anwendung wesentlich praktikabler. ${ }^{19}$ Zudem hat das unbegründete Nebeneinander der Verfahren sowie die ungenaue Formulierung des BGH zur Vorgehensweise bei der Berechnung des Zinsverschlechterungsschadens die unbeabsichtigte Folge, dass die Schadensberechnungen je nach Berechnungsmethode unterschiedlich hoch ausfallen kann. Dies wird in Tabelle 2 anhand eines Beispiels dargestellt: ${ }^{20}$ Dabei werden 3 Zinsszenarien zum

16 J. Luckey: Buch 2: Recht der Schuldverhältnisse, Abschnitt 1: Inhalt der Schuldverhältnisse, in: H. Prütting, G. Wegen, G. Weinreich (Hrsg.): BGB Kommentar, 13. Aufl., Köln 2018, S. 387.

17 BGH Urteil XI ZR 267/96 vom 1.7.1997, S. 9-11 mit Verweis auf BGH Urteil XI ZR 190/90 vom 12.3.1991.

$18 \mathrm{H}$. Schierenbeck, M. Lister, S. Kirmße: Ertragsorientiertes Bankmanagement, Bd. 1, 9. Aufl., Wiesbaden 2014, S. 66 f.

19 Zu den Vorteilen des Aktiv-Passiv-Verfahrens bereits 1998: Vgl. K. Wimmer: Vorfälligkeitsentschädigung und höchstrichterliche Rechtsprechung, Sparkasse, Juli 1998, Nr. 7, S. 326 ff.

20 Vgl. P. Rösler, K. Wimmer, V. Lang, a. a. O., Abschnitt E, S. 169 ff.
Tabelle 2

Berechnung einer Vorfälligkeitsentschädigung nach Aktiv-Passiv- und Aktiv-Aktiv-Methode

in \%

\begin{tabular}{llrrr} 
& Zinsszenario & A & B & C \\
\hline 1 & Ursprünglicher Kreditzins & 3,0 & 3,0 & 3,0 \\
\hline 2 & $\begin{array}{l}\text { Refinanzierungszins bei Kredit- } \\
\text { vergabe }\end{array}$ & 2,0 & 2,0 & 2,0 \\
\hline 3 & Aktueller Kreditzins $t_{0}$ & 1,0 & 0,5 & 1,5 \\
\hline 4 & $\begin{array}{l}\text { Aktueller Refinanzierungs- und } \\
\text { Kapitalmarktzins }{ }_{0}{ }^{1}\end{array}$ & 0,0 & 0,0 & 0,0 \\
\hline $5=1-4$ & Gesamtschaden Aktiv-Passiv & 3,0 & 3,0 & 3,0 \\
\hline $6=1-2$ & Zinsmargenschaden & 1,0 & 1,0 & 1,0 \\
\hline $7=2-4$ & $\begin{array}{l}\text { Korrekter Zins- } \\
\text { verschlechterungsschaden }\end{array}$ & 2,0 & 2,0 & 2,0 \\
\hline $8=6+7$ & $\begin{array}{l}\text { Korrekt berechneter } \\
\text { Gesamtschaden Aktiv-Aktiv }\end{array}$ & 3,0 & 3,0 & 3,0 \\
\hline $9=6$ & Zinsmargenschaden & 1,0 & 1,0 & 1,0 \\
\hline $10=1-3$ & $\begin{array}{l}\text { Falsch berechneter Zins- } \\
\text { verschlechterungsschaden }\end{array}$ & 2,0 & 2,5 & 1,5 \\
\hline $11=9+10$ & $\begin{array}{l}\text { Falsch berechneter Gesamtscha- } \\
\text { den Aktiv-Aktiv }\end{array}$ & 3,0 & 3,5 & 2,5 \\
\hline
\end{tabular}

${ }^{1}$ Die Geld-Brief-Spanne wird hier zur Vereinfachung vernachlässigt.

Quelle: eigene Berechnungen

Zeitpunkt der vorzeitigen Rückzahlung in $\mathrm{t}_{0}$ betrachtet. Zur Vereinfachung wird der aktuelle Kapitalmarktzins jeweils mit derzeit nicht unrealistischen $0,00 \%$ angenommen und lediglich der aktuelle Kreditzins in $t_{0}$ variiert. Wie man der Tabelle 2 in den Zeilen 5 und 8 entnehmen kann, ergibt sich bei korrekter Vorgehensweise bei beiden Methoden, dass der Schaden $3 \%$ beträgt. Nach der Aktiv-Passiv-Methode benötigt man zur Berechnung der Vorfälligkeitsentschädigung lediglich den ursprünglichen Kreditzins (Zeile 1) sowie den Kapitalmarktzins in $\mathrm{t}_{0}$ (Zeile 4 ) aus der Bundesbankstatistik. Nach der Aktiv-Aktiv-Methode ermittelt man den Zinsmargenschaden in Höhe von $1 \%$ aus der Differenz zwischen Kreditzins und Refinanzierungszins zum Zeitpunkt der Vergabe des ursprünglichen Kredits (Zeile 6). Zusätzlich entsteht ein Zinsverschlechterungsschaden in Höhe von $2 \%$ aus der Differenz zwischen Refinanzierungszins und Wiederanlagezins (Zeile 7). Offensichtlich ist diese Methode komplizierter, da als weitere Informationen der Refinanzierungszinssatz bei Kreditvergabe benötigt wird. Der nach der Aktiv-Passiv-Methode berechnete Wert wird also im Rahmen der Aktiv-Aktiv-Methode in zwei Bestandteile zerlegt, ohne dass dies für die Berechnung der Vorfälligkeitsentschädigung notwendig ist. 
Daher ist es auch unverständlich, dass der BGH es erlaubt, im Rahmen der Aktiv-Aktiv-Methode den Zinsmargenschaden auf Basis eines für Banken gleichen Typs üblichen Durchschnittsgewinns zu schätzen und so neue Probleme schafft, da das Rechnen mit der tatsächlichen Marge zu einer Offenlegung interner Betriebsdaten führe. Zudem hat der BGH die Vorgehensweise bei der AktivAktiv-Methode falsch formuliert, sodass es bei wortgetreuer Anwendung je nach Zinskonstellation zu fehlerhaften Berechnungen kommt. Nach BGH soll nämlich der Zinsverschlechterungsschaden durch Vergleich von „Vertragszins und dem Wiederausleihzins“, also dem ursprünglichen und dem aktuellen Kreditzins ermittelt werden. Wie Tabelle 2 zeigt, führt diese Vorgehensweise lediglich dann zum richtigen Ergebnis, wenn Kredit- und Kapitalmarktzinsen sich im Einklang verändern, also die Zinsmarge im Alt- und Neugeschäft konstant bleiben. Dies ist in der Praxis jedoch nicht der Fall. Ist wie in Szenario $B$ die Marge im Neugeschäft in $t_{0}(0,5 \%)$ niedriger als für das ursprüngliche Darlehen (1,0\%), weil der Kreditzins (von 3,0\% auf 0,5\%) stärker gesunken ist als der Kapitalmarktzins (von 2,0\% auf 0,0\%), überzeichnet die Aktiv-Aktiv-Methode den Schaden und umgekehrt. Somit hat die Bank de facto ein Wahlrecht, das für sie jeweils günstigere Verfahren für die Schadensberechnung zu verwenden.

\section{Weitere Ungereimtheiten der rechtlichen Regelungen}

Ob und in welcher Höhe ein Kreditnehmer eine Vorfälligkeitsentschädigung zahlen muss, hängt nicht nur von der Zinsentwicklung ab, sondern auch von der Zinsvereinbarung, dem Darlehenstyp bzw. dem Kreditnehmer sowie den gesetzlichen und vertraglichen Kündigungsrechten. ${ }^{21}$ Die Pflicht zur Zahlung einer Vorfälligkeitsentschädigung kommt nur bei vorzeitiger Rückzahlung festverzinslicher Darlehen in Betracht. Bedeutsam für die Ermittlung der Schadenshöhe ist der vom BGH entwickelte Begriff des Zeitraums der rechtlich geschützten Zinserwartung. ${ }^{22}$ Demnach hat der Kreditgeber bei vorzeitiger Rückzahlung nur für den Zeitraum der Zinsfestschreibung einen Anspruch auf Vorfälligkeitsentschädigung. Spätestens nach Ablauf von zehn Jahren darf der Kreditnehmer jedoch bei Einhaltung einer Kündigungsfrist von sechs Monaten kündigen ( $\$ 489$ Abs. 1 Nr. 2 BGB). Das bedeutet, dass selbst bei einer ursprünglichen Zinsfestschreibung von 15 Jahren ein Kreditnehmer nach zehn Jahren und sechs Monaten vorzeitig ohne Zahlung einer Vorfälligkeitsentschädigung tilgen kann.

21 Vgl. M. Tonner, T. Krüger: Bankrecht 2A, Baden-Baden 2016, S. $191 \mathrm{ff}$. 22 BGH Urteil XI ZR 388/14, S. 11 f vom 19.1.2016.
Zudem reduzieren vertraglich eingeräumte Sondertilgungsrechte die rechtlich geschützte Zinserwartung. Sie sind daher bei der Berechnung der Vorfälligkeitsentschädigung zugunsten des Kreditnehmers zu berücksichtigen. Eine Ausübung von Sondertilgungsrechten kann jedoch in bestimmten Zinskonstellationen für den Kreditnehmer nachteilig wirken. ${ }^{23}$ Daher müsste für jede Sondertilgungsmöglichkeit berechnet werden, ob sie sich positiv oder negativ auf die Höhe der Vorfälligkeitsentschädigung auswirkt. Angesichts einer fehlenden rechtlichen Regelung und aus praktischen Erwägungen heraus schlug die Arbeitsgruppe vor, für eine Vergleichsrechnung stets die frühestmögliche Ausübung in vollem Umfang anzunehmen. Kein Konsens bestand darin, ob man dem Kreditnehmer erlauben sollte, eine für ihn günstigere Ausübung der Sondertilgung darzulegen. ${ }^{24}$ Dabei könnte es durchaus sinnvoll sein, neben der Berechnung der Vorfälligkeitsentschädigung ohne Berücksichtigung der Sondertilgungsrechte, dem Kreditnehmer die Wahl zwischen einer ebenfalls entgeltpflichtigen standardisierten Berücksichtigung und einer vom Kreditnehmer selbst festzulegenden Berücksichtigung der Sondertilgungsrechte zu überlassen. Für die standardisierte Berechnung käme anstelle der Annahme der frühestmöglichen Ausübung sämtlicher Rechte ein Verfahren in Betracht, das die Ausübung der Sondertilgungsrechte nur dann vorsieht, wenn die entsprechende Forward Rate unter dem vereinbarten Kreditzins liegt.

Daneben gibt es gesetzliche Kündigungsrechte, die jedoch nicht von der Zahlung einer Vorfälligkeitsentschädigung bei vorzeitiger Rückzahlung festverzinslicher Kredite entbinden. Für Allgemein-Verbraucherdarlehen gilt allerdings die Besonderheit, dass sie jederzeit und ohne Angabe von Gründen zurückgezahlt werden können, wobei die Vorfälligkeitsentschädigung bei Restlaufzeiten über ein Jahr maximal $1 \%$ und ansonsten maximal $0,5 \%$ des vorzeitig zurückgezahlten Betrags ausmachen darf (§§ 491 Abs.2 und 502 Abs. 3 S. 1 BGB). ${ }^{25}$ Zudem können Kreditnehmer sämtliche Darlehen, die durch Grund- oder Schiffspfandrechte besichert sind ( $\$ 490$ Abs. 2 BGB) sowie festverzinsliche Immobiliar-Verbraucherdarlehen ( 500 Abs. 2 S. 2 BGB) vorzeitig gegen Zahlung einer Vorfälligkeitsentschädigung,

23 Liegt die Forward Rate über dem Kreditzins, ist die Ausübung einer Sondertilgung rein rechnerisch nachteilig für den Kreditnehmer, da er in diesem Fall auf einen höheren Zinssatz verzichtet als er für den Kredit zahlen muss. Vgl. K. Wimmer, P. Rösler: Vorfälligkeitsentschädigung: Kehraus? (1), in: Zeitschrift für Wirtschaft und Bankrecht, 70. Jg. (2016), H. 38, S. 1824 f.

24 Abschlussbericht der Arbeitsgruppe Vorfälligkeitsentschädigung vom 18.9.2018, S. $49 \mathrm{ff}$, S. $63 \mathrm{f}$.

25 Die Deckelung der Vorfälligkeitsentschädigung sowie das Recht zur jederzeitigen Rückzahlung im Falle von Allgemein-Verbraucherdarlehen folgt der Umsetzung der Verbraucherkreditrichtlinie Art. 16 2008/48/EG zum 11.6.2010. Zur Entwicklung des Verbraucherdarlehensrechts G. Nobbe, a.a. O., S. 875 f. 
die nach den vom BGH zugelassenen Verfahren berechnet wurde, zurückzahlen, wenn ein berechtigtes Interesse besteht. ${ }^{26}$ Dies ist etwa dann der Fall, wenn der Kreditnehmer die beliehene Immobilie verkaufen, nicht aber, wenn er auf ein günstigeres Darlehen umschulden möchte. ${ }^{27}$

Schließlich können Kreditverträge auch jederzeit einvernehmlich vorzeitig beendet werden. Hierbei können die Vertragspartner die Vorfälligkeitsentschädigung frei aushandeln, wobei jedoch das Entgelt im Sinne des § 138 BGB nicht sittenwidrig hoch sein darf. ${ }^{28}$ Unverständlich ist, dass Beträge, die je nach Einzelfall zwischen $20 \%$ und $100 \%$ über einer im Falle eines Kündigungsrechts korrekt ermittelten Vorfälligkeitsentschädigung liegen, als rechtlich zulässig betrachtet werden. ${ }^{29}$ Die Pflicht zur Zahlung einer Vorfälligkeitsentschädigung entfällt, wenn der Kreditnehmer beim Darlehensgeber gleichzeitig ein neues Darlehen in übersteigender Höhe zu gleichen oder schlechteren Konditionen aufnimmt oder einen Ersatzdarlehensnehmer stellt, den der Kreditgeber akzeptieren muss. ${ }^{30}$ Dies gilt auch, wenn der Kreditnehmer nach $\S 314$ Abs. 1 BGB aus wichtigem Grund, etwa infolge einer Pflichtverletzung des Kreditgebers, kündigt.

Umgekehrt kam der BGH hingegen zu der Ansicht, dass der Kreditgeber im Falle einer Kündigung bei Verzug des Schuldners, nur den Verzugsschaden, aber keine Vorfälligkeitsentschädigung geltend machen kann, obwohl nach § 314 Abs. 4 BGB ein Recht auf Schadensersatz durch die Kündigung nicht ausgeschlossen wird. ${ }^{31}$ Dabei ignoriert der BGH, dass es sich bei Vorfälligkeitsentschädigung und Verzugsschaden um zwei unterschiedliche Schäden handelt. Dies führt zu dem unbefriedigenden Ergebnis, dass ein säumiger Schuldner, dessen Kredit wegen Verzugs gekündigt wurde, von der Zahlung einer Vorfälligkeitsentschädigung befreit ist, während ein vertragstreuer Schuldner bei vorzeitiger Rückzahlung eine Vorfälligkeitsentschädigung leisten muss. ${ }^{32} \mathrm{Da}$ inzwischen der BGH selbst dargelegt hat, dass der Kreditgeber neben dem Anspruch auf Ersatz des Verzugsschadens auch einen Anspruch auf Vorfälligkeitsentschädigung hat,

26 Zu den Immobiliar-Verbraucherdarlehen zählen beispielsweise auch Immobiliendarlehen ohne grundpfandrechtliche Besicherung.

27 BGH Urteil XI ZR 267/96 vom 1.7.1997, S. 11; G. Nobbe, a. a. O., S. 872

28 BGH Urteil XI ZR 226/02 vom 6.5.2003, S. 7.

29 P. Rösler, K. Wimmer, V. Lang, a.a. O., S. $102 \mathrm{ff}$.

30 G. Nobbe, a.a.O., S. 874 f; BGH Urteil III ZR 197/88 vom 30.11.1989, S. 11; K. Werth: Zweifelsfragen der Vorfälligkeitsentschädigungsberechnung, in: Zeitschrift für Wirtschafts- und Bankrecht, 58. Jg. (2004), H. 9, S. $409 \mathrm{f}$.

31 BGH Urteil XI ZR 103/15 vom 19.1.2016; sowie XI ZR 187/14 vom 22.11.16; zur Kritik daran z. B. G. Nobbe, a. a. O., S. 900; K. Wimmer, P. Rösler, a.a.O., S. $1821 \mathrm{f}$.

32 Zudem gelte diese Entscheidung ohnehin nicht für den Fall, dass die Bank etwa wegen der Verschlechterung der wirtschaftlichen Verhältnisse kündigt. Vgl. K. Wimmer, P. Rösler, a.a. O., S. 1822. wenn er statt zu kündigen Anspruch auf Schadenersatz geltend macht, sollte der Gesetzgeber klarstellen, dass der Kreditgeber auch bei Kündigung Anspruch auf Ersatz beider Schadenskomponenten hat. ${ }^{33}$

Aus den beschriebenen gesetzlichen Regeln ergeben sich weitere Ungereimtheiten, die der Gesetzgeber korrigieren sollte, wobei einige Punkte EU-rechtliche Implikationen haben. Zunächst ist nicht einzusehen, warum Vorfälligkeitsentschädigungen nur im Falle der vorzeitigen Beendigung festverzinslicher Darlehen zu zahlen sind. Immer wieder hat der BGH in seinen Urteilen betont, dass Kreditnehmer einen Anspruch auf Ersatz des entgangenen Gewinns nach $\S 252$ BGB haben. ${ }^{34}$ Dieser Schaden entsteht natürlich auch bei variabel verzinslichen Krediten, da der Kreditnehmer mit der vereinbarten Zinsmarge als Aufschlag auf den Referenzzinssatz über die Vertragslaufzeit rechnet. Dass von einer Minderheit der Arbeitsgruppenmitglieder die grundsätzliche Berechtigung eines Ersatzes für entgangenen Gewinn erneut verneint wurde, ist angesichts des Mangels an stichhaltigen Gegenargumenten unverständlich. ${ }^{35}$

Des Weiteren ist die Berechtigung und Sinnhaftigkeit der Vorzugsbehandlung für Allgemein-Verbraucherdarlehen zu hinterfragen. Die Regelungen widersprechen nämlich den von der damaligen Bundesregierung selbst im Zusammenhang mit der Behandlung von Immobiliar-Verbraucherdarlehen vorgebrachten Behauptungen, dass ein unbeschränktes Kündigungsrecht und eine Deckelung der Vorfälligkeitsentschädigung Festzinskredite verteuerten. ${ }^{36}$ Dabei ist allerdings nur letzteres als stichhaltig anzusehen, da ein unbeschränktes Kündigungsrecht zumindest dann nicht zu einer Verteuerung führt, wenn der Kreditgeber durch die Zahlung einer Vorfälligkeitsentschädigung wirtschaftlich so gestellt wird, wie wenn das Darlehen vertragsgemäß bis zum Ende der Zinsfestschreibung bedient worden wäre.

Daraus folgt, dass man, auch im Sinne der Verbraucher, die beschriebene Sonderbehandlung aufgeben und stattdessen allen Kreditnehmern ein unbeschränktes Kündi-

33 BGH Urteil XI-ZR 445-17 vom 20.2.2018.

34 Vgl. unter anderem BGH Urteil XI ZR 190/90 vom 12.3.1991; BGH Urteil VIII ZR 317/93 vom 29.6.1994.

$35 \mathrm{Vgl}$. Arbeitsgruppe Vorfälligkeitsentschädigung, a.a.O., S. 23 f. So wurde behauptet, dass erst die vorzeitige Rückzahlung des Kredits der Bank angesichts knapper Ressourcen ein Neugeschäft ermögliche. Ebenso abwegig die Behauptung, dass ein Schadenersatz nicht gerechtfertigt sei, da bei vorzeitiger Ablösung eines Darlehens infolge eines Verkaufs typischerweise ein anderer Käufer ein Darlehen - wenn auch bei einer anderen Bank - aufnehme und so volkswirtschaftlich gesehen kein Margenschaden entstehe. Vgl. Arbeitsgruppe Vorfälligkeitsentschädigung, S. 23 f. Diese Argumentation wurde vom BGH bereits in XI ZR 267/96 vom 1.7.1997 zu Recht zurückgewiesen.

36 Deutscher Bundestag: Drucksache 18/5922, Entwurf eines Gesetzes zur Umsetzung der Wohnimmobilienkreditrichtlinie vom 7.9.2015 S. $90,92 \mathrm{f}$. 
gungsrecht gegen Zahlung einer angemessenen Vorfälligkeitsentschädigung einräumen sollte, zumal der Anspruch auf eine vorzeitige Kreditabwicklung aus berechtigtem Interesse ohnehin sehr weit ausgelegt wird. ${ }^{37}$ Eine solche Regelung würde zudem Gerichte entlasten, da weder die Frage der Berechtigung einer Kündigung noch die Frage nach der Sittenwidrigkeit von Aufhebungsentgelten untersucht werden müsste. ${ }^{38}$

\section{Fazit}

Die Untersuchung zeigt, dass zumindest folgende Aspekte der gesetzlichen Regeln und der vom BGH entwickelten Praxis kritikwürdig sind und daher Ansatzpunkte für entsprechende gesetzliche Änderungen bieten:

- Statt des derzeitigen Nebeneinanders von zwei bzw. drei vom BGH entwickelten Berechnungsmethoden sollte die Aktiv-Passiv-Methode rechtsverbindlich als einzig zulässiges Verfahren vorgeschrieben werden.

- Die für die Berechnung nach der Aktiv-Passiv-Methode notwendigen Parameter sollten rechtsverbindlich vorgegeben werden.

- Die für die Berechnung des Barwerts notwendigen Zerobondabzinsungsfaktoren werden bereits von der Deutschen Bundesbank täglich veröffentlicht.

- Die Risikokosten könnten je nach Kreditart als Durchschnittswerte für die gesamte Branche aus den von den Banken für die Eigenmittelunterlegung ermittelten $\mathrm{Pa}$ rametern PD und LGD abgeleitet und ebenfalls von der Bundesbank oder BaFin zur Verfügung gestellt werden.

- Die ersparten Verwaltungskosten p.a. und die für die Berechnung einer Vorfälligkeitsentschädigung erfor-

37 BGH Urteil XI ZR 267/96 vom 1.7.1997, S. 11.

38 K.-O. Knops: Nichtabnahme- und Vorfälligkeitsentschädigung, in: P. Derleder et al. (Hrsg.): Deutsches und europäisches Bank- und Kapitalmarktrecht, Berlin, Heidelberg 2017, S. 804 ff; K. Wimmer, P. Rösler: Vorfälligkeitsentschädigung bei vorzeitiger Beendigung von Darlehensverträgen, in: Zeitschrift für Wirtschafts- und Bankrecht, 59. Jg. (2005), H. 40, S. 1880. derlichen Bearbeitungsentgelte könnten etwas unterhalb der derzeit akzeptierten Werte pauschal festgelegt und gegebenenfalls jährlich an die Gehaltsentwicklung angepasst werden.

- Die unterschiedliche Behandlung nach Zinsfestschreibung und Darlehenstyp bzw. Kreditnehmer sollte zugunsten eines einheitlichen Verfahrens mit folgenden Konsequenzen aufgegeben werden:

- Kreditnehmer sollten Kredite gegen Zahlung einer Vorfälligkeitsentschädigung jederzeit ablösen dürfen.

- Aufhebungsentgelte sollten der Höhe der Vorfälligkeitsentschädigung entsprechen, was bei einem uneingeschränkten Kündigungsrecht gegen Vorfälligkeitsentschädigung automatisch gegeben wäre.

- Kreditnehmer sollten das Recht auf einen Vorfälligkeitsnutzen erhalten.

- Kreditgeber sollten eine Vorfälligkeitsentschädigung erhalten, die unabhängig vom Darlehenstyp bzw. Kreditnehmer und ohne gesetzliche Obergrenze, wie derzeit bei Allgemein-Verbraucherdarlehen vorgesehen, berechnet wird.

- Kreditgeber sollten auch bei der vorzeitigen Ablösung eines variabel verzinslichen Darlehens eine Vorfälligkeitsentschädigung als Ausgleich für den entgangenen Margenschaden erhalten.

- Kreditgeber sollten auch bei Kündigung infolge von Pflichtverletzung durch den Kreditnehmer neben dem Recht auf Ausgleich des Verzugsschadens auch ein Recht auf Vorfälligkeitsentschädigung haben.

Obwohl dieses Maßnahmenpaket sich um einen ausgewogenen Interessenausgleich zwischen Kreditnehmern und Kreditgebern bemüht, dürften die Hürden für eine Umsetzung angesichts der Erklärungsbedürftigkeit, der Ressentiments gegenüber Banken und der EU-rechtlichen Implikationen sehr hoch sein.

Title: Prepayment Penalty: Balancing of Interests Pragmatically is Feasible

Abstract: Disputes about prepayment penalties have been keeping German courts busy for many years. This is partly because the German government has refrained to date from deciding on a legally binding method for the computation. Two federal ministries installed a working group, which presented its results at the end of 2018. However, the group was able to agree upon neither the methodology nor on improving the transparency of the computation. Whether and how the government will respond remains unclear. Therefore, this paper will describe the method of computation, investigate the most controversial subjects and present a few pragmatic solutions for further discussion.

JEL Classification: G210 